\title{
Retinal nerve fiber layer abnormalities in Alzheimer's disease
}

\author{
Thomas R. Hedges III ${ }^{1,2}$, Rosalba Perez Galves ${ }^{3}$, \\ Donna Speigelman ${ }^{4}$, Nancy R. Barbas ${ }^{1,2,5}$, Eli Peli ${ }^{1}$ and \\ Christine J. Yardley ${ }^{1}$
}

Departments of Ophthalmology ${ }^{1}$, Neurology ${ }^{2}$, Tufts University, New England Medical Center, Boston, Massachusetts; Center for Studies of Sensory Impairment, Aging and Metabolism ${ }^{3}$, Hospital de Ojos y Oidos 'Dr. Roldolfo Robles V., Guatemala City, Guatemala;

Department of Epidemiology ${ }^{4}$, Harvard School of Public Health, Boston, Massachusetts, and University of Michigan Medical Center,

Department of Neurology ${ }^{5}$, Ann Arbor, Michigan

\begin{abstract}
Retinal nerve fiber layer (RNFL) photographs from $26 \mathrm{pa}-$ tients with Alzheimer's disease and 23 normal, age-matched, control subjects were reviewed for quality and abnormalities by two observers. A higher proportion of Alzheimer's patients showed RNFL abnormalities when compared to control subjects. There was some disagreement between the two observers regarding quality and frequency of abnormalities, reflecting suboptimal quality of the photographs obtained in patients with advanced Alzheimer's disease. Although these findings add to the clinical and histopathological evidence that ganglion cell degeneration occurs in Alzheimer's disease, the difficulty in obtaining and evaluating retinal nerve fiber layer photographs, especially in advanced cases, may limit the clinical usefulness of retinal nerve fiber layer analysis in such patients.
\end{abstract}

Key words: Alzheimer's disease - retinal nerve fiber layer - optic neuropathy - aging - vision.

Acta Ophthalmol. Scand. 1996: 74: 271-275

$\mathrm{T}$ here has been continued interest and some controversy regarding the visual effects of Alzheimer's disease. Although it is clear that many of the visual problems associated with Alzheimer's disease are due to cortical dysfunction (Schlotterer et al. 1983; Cogan 1985, Kiyosawa et al. 1989; Mendez et al. 1990; Cronin-Golomb et al. 1991; Levine et al. 1993; Hutton et al. 1993) and, although eye movement abnormalities may play a role (Feldon nerve function, such as visual field and color vision testing, as well as visual evoked potential recording and observation of optic disc color and contour (Cogan 1985; Cronin-Golomb et al. 1991; Sadun et al. 1987; Nissen et al. 1985; Rizzo et al. 1992; Katz et al. 1989). Clinical evaluation of the retinal nerve fiber layer has been shown to be an excellent way to detect and measure the effects of a variety of diseases on the optic nerve (Quigley et al. 1982; Peli et al. 1987). So far, only one study, which was primarily directed toward analysis of the appearance of the optic nerve head, has included evaluation of the clinical appearance of the retinal nerve fiber layer (Tsai et al. 1991). Photographs of variable quality, from 24 patients with Alzheimer's disease reviewed by one reader, showed a significant number of abnormalities, indicating the need for further study in this area. We evaluated the retinal nerve fiber layer in a group of $\mathrm{Alz}$ heimer's patients in various stages of Alzheimer's disease, including later stages. To control for quality of photographs, two observers were asked to review photographs in a masked fashion allowing for comparison of intra- and inter-observer variability. 


\section{Patients, Subjects and Methods}

\section{Normal subjects}

Photographs from 19 normal subjects were collected. These individuals had no evidence of glaucoma, nutritional, or other optic nerve disease. Clinically normal eyes from two patients with contralateral anterior ischemic optic neuropathy, and the normal eyes from two patients with optic neuropathy from compression of the contralateral optic nerve (aneurysm in one and optic nerve sheath meningioma in the other) were also used. All normal subject eyes had normal Goldmann or automated visual fields (Octopus 2000, Interzeag), color vision, acuity, intraocular pressures and clinical examination, except for lens changes associated with age. Ages in the control group range from 52-93 years with a mean of 71 years.

\section{Alzheimer's patients}

Forty-seven patients were evaluated for the study. All patients satisfied the National Institutes of Health and $\mathrm{Alz}^{-}$ heimer's Disease and Related Disorders Association (NINCDS) ADRDA) criteria for the diagnosis of probable Alzheimer's disease (McKhann et al. 1984). All patients were, or continue to be, part of other studies of Alzheimer's disease at either Tufts University (New England Medical Center, Boston, Massachusetts), Harvard University (Brigham and Women's Hospital, Boston, Massachusetts), or Yale University (Jewish Home for the Elderly, Fairfield, Connecticut). Records were thoroughly reviewed to exclude patients with nutritional deficiency, focal neurologic disease (such as stroke), and alcoholism. All patients had computed tomography or magnetic resonance imaging which showed no evidence of focal neurologic disease. All patients had previous eye examinations showing no evidence of glaucoma, senile macular degeneration, other optic neuropathies (such as anterior ischemic optic neuropathy), or retinal artery occlusions.

Because different types of mental status examinations had been per- formed on different patients, a separate dementia scale was created.

Stage 1: Mild memory/language, behavioral difficulties, able to care for self.

Stage 2: Moderate memory/lan guage, behavioral difficulties, but requiring constant supervision.

Stage 3: Severe dementia, some language function remaining, able to follow simple commands.

Stage 4: Complete loss of language and ability to ambulate. Unable to follow commands.

Ages of patients with Alzheimer's disease ranged from 54 to 93 years with a mean of 73 years.

\section{Ophthalmic examination}

All patients were evaluated for visual acuity when possible. Visual fields were usually performed by Goldmann perimetry, and by confrontation in patients in the later stages of the disease. Color vision using American Optical Hardey Ritter Rand color plates was performed on cooperative individuals. All patients had their refractive errors assessed by either standard refraction techniques or retinoscopy. All patients had intraocular pressure measured using applanation tonometry. Slitlamp examinations were performed on all cooperative patients to rule out significant cataract. Dilated funduscopic examination was performed on all individuals.

\section{Photographic techniques}

All photographs were taken either with a Canon CF-60Z or a Zeiss FK 30 fundus camera. A SE-4 blue fluorescein excitor filter was used in patients without significant cataract, and an SE540 , green, interference filter was used for patients with mild cataract. Color photographs were obtained using $\mathrm{Fu}$ gichrome slide film. All red-free, black and white photographs were taken using Panatomic X film, using 30 degree fields of four quadrants around the optic disc, attempting to obtain at least two photographs per quadrant. Between 10-30 photographs per eye were taken on all patients.

\section{Photograph selection}

Of the 47 patients entered in the study, 34 patients were cooperative enough for photography. However, prelimi- nary analysis of the photographs showed that those from eight patients with Alzheimer's disease were determined to be too poor or too few for analysis. This left photographs from 26 Alzheimer's patients and 23 control subjects for further analysis for both quality and abnormalities.

\section{Photograph analysis}

In order to standardize material for each patient, and because of the variable number of photographs taken for each patient, it was decided that a masked analysis should include two observers using the best quality photographs available. A preliminary analysis for retinal nerve fiber layer defects showed that, of 15 patients who were found to have abnormalities, 14 had abnormalities in both eyes, giving an inter-eye correlation of 0.99 . It was decided that the best photographs of one eye from each patient and each normal subject would be shown to one observer on two occasions and to another observer on another occasion. Following this, all slides from Alzheimer's patients and all control subjects were made available to one observer for unmasked analysis.

During the first reading, by observer 1 (TRH), the best photograph from each quadrant from the slides selected from 26 Alzheimer's patients randomly mixed with slides from 23 control subjects were shown in a masked fashion. A second reading was performed by observer 1 (TRH 2), this time using photographs from the superotemporal quadrant from $26 \mathrm{pa}-$ tients with Alzheimer's disease and 19 control patients. Observer 2 (EP) was shown superotemporal photographs from the same 26 Alzheimer's patients and 19 normal subjects. An additional reading of all photographs from both eyes of 26 Alzheimer's patients and 23 normal subjects were reviewed in an unmasked fashion by observer 1 .

The observers identified the photographs as normal (Fig. 1), abnormal, or unreadable. Abnormal photographs were further qualified as having either diffuse nerve layer drop-out, slit defects, or wedge defects. Diffuse dropout was determined by loss of striations on the surface of the retina combined with prominence of small blood vessels running perpendicular to the 


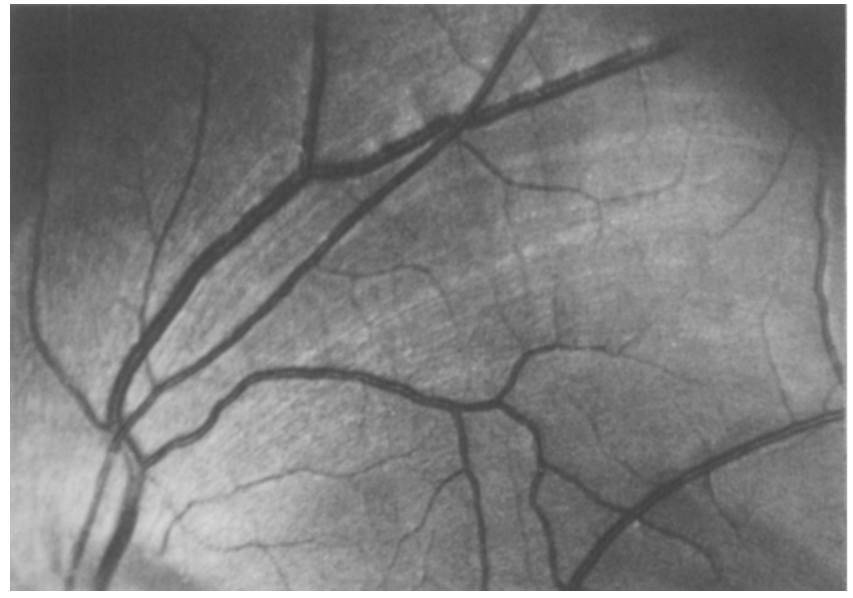

Fig. 1. Inferior temporal quadrant of a left fundus showing normal retinal nerve fiber layer striations.

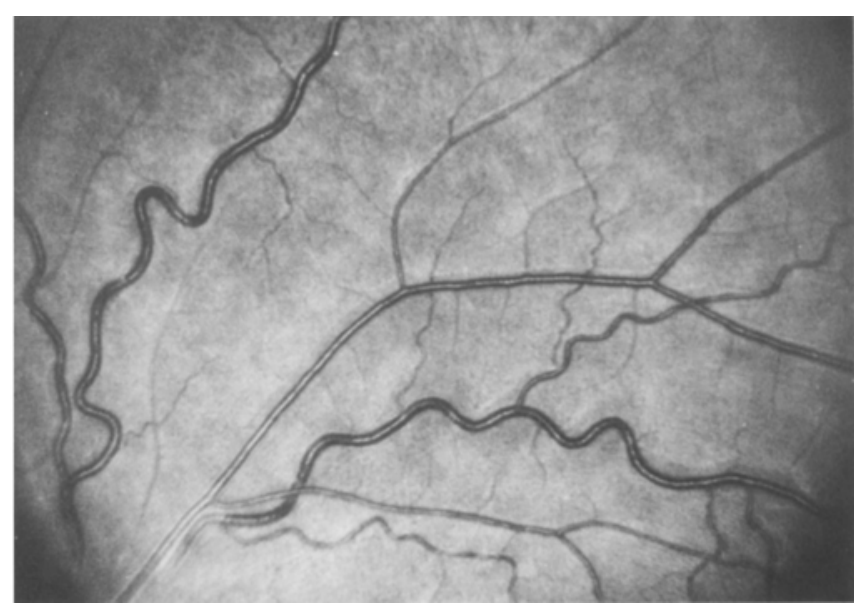

Fig. 2. Superior temporal quadrant of a left fundus showing diffuse loss of the retinal nerve fiber layer with exposure of blood vessels. nerve fiber layer throughout the photograph (Fig. 2). Slit defects were identified as dark lines running parallel to normal nerve fiber striations extending from the optic disc for a distance of at least 2 optic disc diameters (Fig. 3). Wedge defects were determined as slit defects greater than the width of a large vein $(200$ microns).

\section{Statistical analysis}

Odds ratios and $95 \%$ confidence intervals for RNFL abnormalities determined for each observation were calculated from logistic regression model (Homer \& Lemeshow 1989), of the presence or absence of RNFL abnormalities relative to age, and stage of Alzheimer's disease. Inter- and intraobserver comparisons were made by determining kappa statistics and percent concordance. This was done with regard to differences which occurred in determinations of quality of photo- graphs as well as abnormalities detected by the two observers (inter-observer agreement), as well as abnormalities detected by the same observer on different occasions (intra-observer agreement). For most purposes, kappa values greater than 0.75 may be considered to represent excellent agreement beyond chance, and values below 0.40 may be considered to represent poor agreement (Fleiss 1981).

\section{Results}

\section{Staging of Alzheimer's disease}

Of those individuals determined to have readable photographs by observer 1, after the first observation, the severity of Alzheimer's disease was as follows: 10 patients were placed in stage 1,10 patients in stage 2,3 patients in stage 3 , and 2 patients in stage 4. Of these 25 patients, 12 had mini

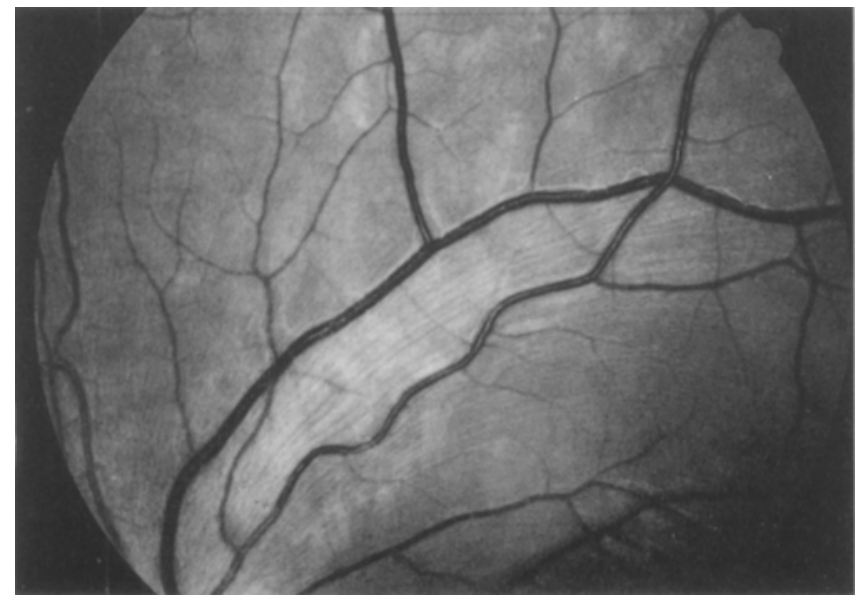

Fig. 3.

Superior temporal quadrant of a left fundus showing several slit defects in the retinal nerve fiber layer. mental status examinations. Although there was some overlap in test scores, the mean test scores among the patients were as follows: in stage 1,21.0, in stage $2,15.8$, in stage $3,5.5$, and in stage 4,0 .

\section{Neuro-ophthalmic findings}

Neuro-ophthalmic symptoms described by some patients, included difficulty reading in 2 individuals, visual spatial disorientation in 2 patients, and visual hallucinations in 1 subject. Clinical evaluation showed optic disc pallor in 1 patient. Visual field defects using Goldmann perimetry are found in none of 13 patients tested. Color vision defects were found in 3 of 7 patients tested, and color naming difficulty was noted in 1 of the 7 patients tested. An abnormal pattern evoked potential was obtained in 1 of 9 patients tested, and one individual had a normal pattern evoked potential but an abnormal flash evoked potential. Ocular motor findings included abnormal saccades in 4 of 26 patients, delayed re-fixation in 8 of 26 patients and pursuit deficits in 18 of 26 patients.

\section{Retinal nerve fiber layer abnormalities} Findings by observer 1 (both masked readings), observer 2 , (single masked reading) and observer 1 (unmasked reading) are shown in Table 1 . Odds ratios for unmasked observations range from 4.1 to 11.2. Observer 2 did not see a significant difference between Alzheimer's patients and normal subjects. However, review of all available photographs from both Alzheimer's patients 
Table 1. (Nl: normal. Abn: abnormal. Rej: reject).

\begin{tabular}{|c|c|c|c|c|c|c|c|c|}
\hline & \multicolumn{3}{|c|}{ Control subjects } & \multicolumn{3}{|c|}{ Alzheimer's patients } & \multirow{2}{*}{$\begin{array}{l}\text { Odds } \\
\text { ratio }\end{array}$} & \multirow{2}{*}{$\begin{array}{l}95 \% \text { confidence } \\
\text { interval }\end{array}$} \\
\hline & $\mathrm{Nl}$ & Abn & Rej & $\mathrm{Nl}$ & Abn & Rej & & \\
\hline TRH-1 & 18 & 3 & 2 & 12 & 13 & 1 & 6.50 & $1.3-36.7$ \\
\hline TRH-2 & 14 & 1 & 4 & 10 & 8 & 8 & 11.20 & $1.06-279.0$ \\
\hline EP & 11 & 3 & 5 & 8 & 9 & 9 & 4.13 & $0.67-28.1$ \\
\hline TRHunmasked & 19 & 2 & 2 & 13 & 11 & 1 & 8.04 & $1.36-\quad 3.2$ \\
\hline
\end{tabular}

and controls including both eyes (unmasked) showed an odds ratio of 8.0 $(\mathrm{p}<0.01,95 \%$ confidence interval 1.3 63.2).

Types of nerve fiber layer abnormalities based on observations by the first observer of 13 patients with abnormalities includes diffuse nerve fiber layer drop-out ( 10 patients), slit defects ( 1 patient), and diffuse nerve fiber layer drop-out plus slits (2 patients).

\section{Retinal nerve fiber layer abnormalities and stage of disease}

Using a logistic regression analysis of presence or absence of RNFL abnormalities on stage of the disease and age, a trend towards increasing abnormalities with progressive stages of the disease occurred. This trend was not statistically significant (Fig. 4 - histogram).

\section{Inter-observer and intra-observer comparisons}

Comparisons were done between different observations for quality of photographs. Comparing readings from observer 1 , the percent concordance of intra-observer agreement was $73.5 \%$ for Alzheimer's patients and control subjects combined (kappa = 0.17 for Alzheimer's patients, 0.31 for controls, and 0.23 for both). Inter-ob-

server agreement between the second reading by observer 1 and the reading by observer 2 , was $68.8 \%$ for Alzheimer's patients and control subjects combined (kappa $=0.14$ for Alzheimer's patients, 0.25 for controls and 0.19 for both)

The intra-observer concordance for assessment of abnormalities, observer 1 , first analysis vs second analysis was 96.9\% (kappa $=0.93$ ). Percent concordance for inter-observer analysis for abnormalities (observer 1 (first reading) vs. observer 2 ) was $76.7 \%$ $(\mathrm{kappa}=0.58)$.

\section{Discussion}

Several studies of visual function in patients with Alzheimer's disease indicate that there is a problem with central processing of visual information in affected individuals (Schlotterer et al. 1983; Cogan 1985; Kiyosawa et al. 1989; Mendez et al. 1990; Cronin-Golopmb et al. 1991; Levine et al. 1993; Hutton et al. 1993). However, there has been increasing evidence of involvement of the anterior visual pathways, especially since Hinton et al. found reduced numbers of ganglion cells and axons in an autopsy study of patients with Alzheimer's disease (Hinton et al. 1986). More recently, it has been sug-

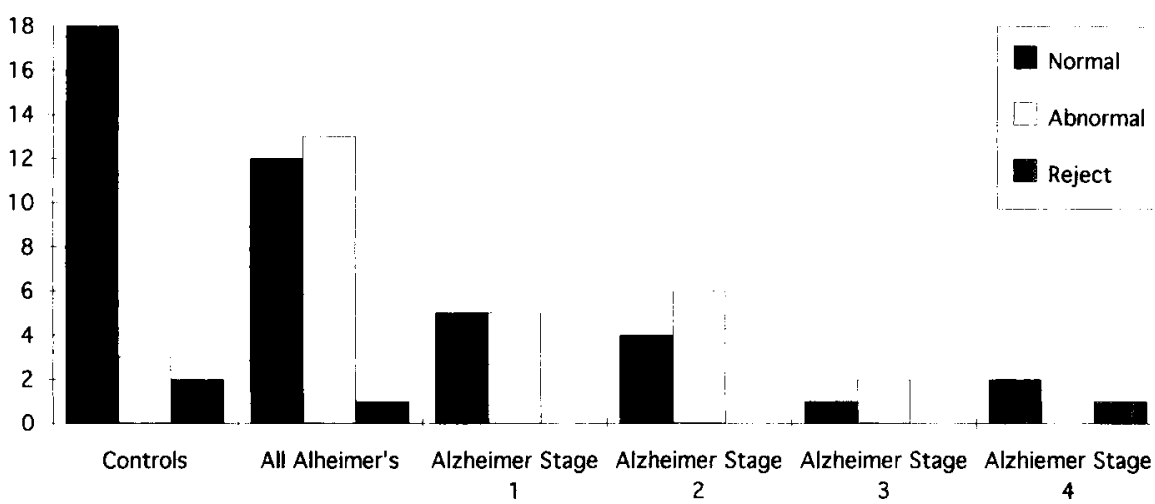

Fig. 4. Retinal nerve fiber layer findings: Alzheimer's vs control subjects (TRH-I). gested that large, M-cell axons may be the first to degenerate in Alzheimer's disease (Sadun \& Bassi 1990). Clinical evidence for optic neuropathy in Alzheimer's disease has been provided by several studies (Sadun \& Bassi 1990; Katz et al. 1989), although others have found no evidence of significant optic nerve changes in Alzheimer's disease (Cronin-Golomb et al. 1991; Rizzo et al. 1992). Only one study has included specific evaluation of the retinal nerve fiber layer (RNFL) in patients with Alzheimer's disease (Tsai et al. 1991). The authors of this study found a higher proportion of RNFL abnormalities among 26 patients with Alzheimer's disease as compared to 32 control patients when examined with red free photography. Although the pallor-area to disc-area ratio was not significantly different between patients with Alzheimer's disease and controls the Alzheimer's patients with higher pallor-area to disc-area as well as those with increased cup-disc ratios and increased cup volume and decreased disc-rim areas had higher Alzheimer's disease assessment scale scores. In the same study, of 26 patients photographed, 22 had red free, RNFL photographs of high enough quality for evaluation. Of these, 5 were found to have normal RNFL. The numbers of photographs per patient and the specific problems regarding quality of photographs were not noted (Schlotterer 1983).

In the present study, we also had problems with the number and quality of photographs. It was recognized early in the study that this would be a problem primarily because patients with Alzheimer's disease are less cooperative for photography, and, because they are more likely to have agerelated lens opacities. Therefore, we decided to incorporate into the study a mechanism for evaluation of variability in photographic evaluation in order to determine if our findings were reliable. Before reviewing all photographs available, selected photographs (the best single photograph available for each patient) were shown to one observer on two occasions to calculate inter-observer variability, and to a second observer to calculate intra-observer variability. Furthermore, comparisons of photographic quality as well as 
incidence of RNFL abnormalities were made. The concordance for intraobserver agreement of $73.5 \%$ and the inter-observer agreement of $68.8 \%$ for quality may reflect the overall, less than optimal, quality of the photographs. The intra-observer agreement for RNFL abnormalities of $96.9 \%$ and the inter-observer agreement for RNFL abnormalities of $76.7 \%$ may have been related to the greater number of photographs rejected by observer 2 .

Whether our findings, and those of others will be clinically useful in the diagnosis and treatment of Alzheimer's disease remains to be seen. Furthermore, our findings do not help determine the extent to which anterior visual pathway changes in Alzheimer's disease contribute to the central abnormalities of visual function found in these individuals. Although we could not demonstrate a definite increase in the frequency of RNFL abnormalities with increasing severity or duration of Alzheimer's disease in our patients, because of the low number of individuals in the very late stages of the disease, our findings and those of Tsai et al. (1991) suggest that optic nerve degeneration with Alzheimer's disease is progressive. To the extent that abnormalities of the RNFL could be associated with earlier or later mortality, any study including only survivors, could be biased. A longitudinal study of RNFL findings in a cohort of early Alzheimer's patients followed throughout their course would be needed to determine the relationship between RNFL abnormalities and Alzheimer's disease and to determine if detection and monitoring of RNFL changes will be of use in diagnosis or prognosis as well as determining response to treatment of patients with Alzheimer's disease.

\section{Acknowledgment}

Supported in part by the Alzheimer's Disease and Related Disorders Association.

\section{References}

Cogan DG (1985): Visual disturbances with focal progressive dementing disease. AJO 100: 68-72.
Cronin-Golomb A, Corkin S, Rizzo JF, Cohen J, Growdon JH \& Banks KS (1991): Visual dysfunction in Alzheimer's disease: Relation to normal aging. Ann Neurol 29:4152.

Feldon SE \& Langston JW (1977): Squarewave jerks: a disorder of micro-saccades. Neurology 27: 278-281.

Fleiss JL (1981): Statistical methods for rates and proportions, 2 nd Edition, p. 225. John Wiley \& Son, New York.

Fletcher WA \& Sharpe JA (1988): Smooth pursuit dysfunction in Alzheimer's disease. Neurology 38: 272-277.

Hinton DR, Sadun SA, Blanks JC \& Miller CA (1986): Optic-nerve degeneration in Alzheimer's disease. N Engl J Med 315: 485-487.

Homer DW\& Lemeshow S (1989): Applied logistic regression. John Wiley \& Son, New York.

Hutton JT, Morris JL, Elias JW \& Poston JN (1993): Contrast sensitivity dysfunction in Alzheimer's disease. Neurology 43: 2328-2330.

Katz B, Rimmer S, Iragui V \& Katzman R (1989): Abnormal pattern electro-retinogram in Alzheimer's disease: Evidence for retinal ganglion cell degeneration? Ann Neurol 26: 221-225.

Kiyosawa M, Bosley TM, Chawluk J, Jamieson D, Shatz NJ, Sauino PJ, Sergott RC, Reivich M \& Alavi A (1989): Alzheimer's disease with prominent visual symptoms. Clinical and Metabolic Evaluation. Ophthalmology 96: 1077-1086.

Levine DN, Lee JM \& Fisher CM (1993): The visual variant of Alzheimer's disease: A clinicopathologic case study. Neurology 43: 305-313.

McKhann G, Drachman D, Folstein M, Katzman R, Price D \& Stadlan EM (1984): Clinical diagnosis of Alzheimer's disease: Report of the NINCDS-ADRDA work group* under the auspices of the Department of Health and Human Services Task Force on Alzheimer's disease. Neurology 34: 939-944.

Mendez MF, Tomsak RL \& Remler B (1990): Disorders of the visual system in Alzheimer's disease. J Clin Neuro-ophthalmol 10:62-69.

Nissen MJ, Corkin S, Buonanno FS, Growdon JH, Wray SH \& Bauer J (1985): Spatial vision in Alzheimer's disease. General findings and a case report. Arch Neurol 42: 667-671.

Peli E, Hedges TR III, McInnes T Hamlin J \& Schwartz B (1987): Nerve fiber layer photography. A comparative study. Acta Ophthalmol (Copenh) 65:71-80.

Quigley HA, Addicks EM \& Green WR (1982): Optic nerve damage in human glaucoma. III. Quantitative correlation of nerve fiber loss and visual field defect in glaucoma, ischemic neuropathy, papilledema, and toxic neuropathy. Arch Ophthalmol 100: 135-146.

Rizzo JF, Cronin-Golomb A, Growdon JH, Corkin S, Rosen TJ, Sandberg MA, Chiappa KH \& Lessel S (1992): Retinocalcarine function in Alzheimer's disease. A clinical and electrophysiological study. Arch Neurol 49: 93-101.

Sadun AA \& Bassi CJ (1990): Optic nerve damage in Alzheimer's disease. Ophthalmology 97: 9-17.

Sadun AA, Borchert M, DeVita E, Hinton DR \& Bassi CJ (1987): Assessment of visual impairment in patients with $\mathrm{Alz}$ heimer's disease. AJO 104: 113-120.

Schlotterer G, Moscovitch M \& CrapperMcLachlan D(1983): Visual processing deficits as assessed by spatial frequency contrast sensitivity and backward masking in normal ageing and Alzheimer's Disease. Brain 107: 309-325.

Tsai CS, Ritch R, Schwartz B, Lee SS, Miller NR, Chi T \& Hsieh FY (1991): Optic nerve head and nerve fiber layer in Alzheimer's disease. Arch Ophthalmol 109: 199-204.

Received on October 10th, 1995.

Corresponding author:

Thomas R. Hedges III, MD

New England Medical Center

750 Washington Street

Box 381

Boston, Massachusetts 02111 . 